\title{
The fine tuning problem revisited in the light of the Taylor-Lagrange renormalization scheme.
}

\section{P. Grangé, ${ }^{a}$ J-F Mathiot, ${ }^{b}$ Bruno Mutet $^{* a}$ and E. Werner ${ }^{c}$}

${ }^{a}$ Laboratoire de Physique Théorique et Astroparticules, Université Montpellier II, CNRS/IN2P3

Place E. Bataillon, F-34095 Montpellier cedex 5, France

${ }^{b}$ Laboratoire de Physique Corpusculaire, Université Blaise Pascal, CNRS/IN2P3

24 avenue des Landais, F-63177 Aubière Cedex, France

${ }^{c}$ Institut für Theoretische Physik, Universität Regensburg

Universitätstrasse 31, D-93053 Regensburg, Germany

\begin{abstract}
The radiative corrections to the Higgs mass are calculated to the first loop order using both the usual sharp cut-off procedure and Taylor-Lagrange renormalization scheme (TLRS). Results appear to differ significantly. The TLRS results allow for a different interpretation of the Higgs renormalized mass. The fine tuning issue is discussed.
\end{abstract}

Light Cone 2010: Relativistic Hadronic and Particle Physics

June 14-18, 2010

Valencia, Spain

\footnotetext{
*Speaker.
} 


\section{Introduction}

The standard model of particles physics is commonly thought of as an effective theory valid up to some energy scale $\Lambda_{e f f}$. We know that at most $\Lambda_{e f f}<M_{P}$, where $M_{P}$ is the Planck mass, $\left(\simeq 10^{19} \mathrm{GeV}\right)$ since gravitational effects become relevant, and cannot be neglected at that scale. We thus consider the standard model lagrangian as the effective low energy part of a more general and unknown lagrangian describing the underlying theory. Even if it is not a parameter of the standard model Lagrangian, $\Lambda_{\text {eff }}$ should be taken in consideration in our calculations. Namely, all typical intrinsic momenta which are relevant for the description of any physical process should be less or equal to $\Lambda_{\text {eff }}$. At tree level, these momenta are defined by the typical kinematical variables of the process, like $\sqrt{Q^{2}}$ in Deep Inelastic Scattering (DIS). They are thus under complete control. However, beyond tree level, one has to deal with intrinsic momenta in loop contributions, that may be large. To adress the problem of loop calculations, we have to consider renormalization theory. There are various renormalization types at hand. Among others we can cite [1]: the bare multiplicative renormalization, the counterterms method and the BPHZ method. For the sake of simplicity, we will be using the first one as we will be interested in the definition of the renormalized mass alone.

To be able to handle divergent quantities, such as loop integrals, one has to pick up a regularization scheme. Two regularization methods will be used in this study. The first one exhibits a very large mass scale, denoted by $\Lambda_{C}$. This mass scale is either a naïve cut-off in (Euclidean) four-momentum space, or the mass of Pauli-Villars (PV) particles in its covariant version, the PV regularization scheme. This explicit mass scale should be much larger then any typical energy, or momentum scale relevant in the calculation of the theoretical physical amplitude. The second one, wich we will focus on, is the Taylor-Lagrange regularization scheme (TLRS). This scheme, wich has been developped recently [2,3], is based on the Epstein-Glaser [4] procedure to define physical amplitudes in terms of operator valued distributions (OPVD) acting on test functions. All amplitudes are then completely finite from the start, and depend on an arbitrary, finite, dimensionless scale $\eta^{2}$. The connection of the Epstein-Glaser formalism with the BPHZ renormalization scheme has been shown in Ref. [5]. The TLRS will be presented in more details in Sec. 2.

Using a naïve cut-off to regularize the bare amplitudes, the radiative corrections to the Higgs mass immediately lead to a quadratic dependency of the regulated amplitudes on the cut-off scale $\Lambda_{C}$. The (square of the) physical mass, $M_{H}$, defined at the pole of the two-body Green's function, can be schematically written as

$$
M_{H}^{2}=M_{0}^{2}+b \Lambda_{C}^{2}+\ldots
$$

where $M_{0}$ is the bare mass of the Higgs particle, and $b$ is a combination of the top quark, $W, Z$ bosons and Higgs masses. The so-called fine-tuning problem arises from the requirement that $\Lambda_{C}$ should be much larger than any typical energy scale relevant for the description of the theoretical physical amplitude but still smaller than $\Lambda_{e f f}$. If one believes $M_{H}$ to be about a few hundreds of $\mathrm{GeV}$, a large cancellation between $M_{0}^{2}$ and $b \Lambda_{C}^{2}$ should be enforced by hand, hence the name finetuning.

However, when using the TLRS, the situation is quite different. Indeed, as the TLRS yields completely finite amplitudes no such problem appears. To show how this work in practice,we will, in 
section 3, first calculate the radiative corrections to the Higgs mass to the first loop order using alternatively a sharp cut-off procedure and the TLRS and then compare and discuss both results.

\section{The Taylor-Lagrange renormalization scheme}

This scheme is based on the observation that any quantum field theory is an OPVD [6]. This has been known for a long time. However, its full significance for practical calculations was not fully recognized until recently $[2,3,4,5,8]$.

As any distribution, quantum fields should be defined by their application on test functions, denoted by $\rho$, with well identified mathematical properties [9]. In flat space, the physical field $\varphi(x)$ is thus defined by [2]

$$
\varphi(x) \equiv \int d^{D} y \phi(y) \rho(x-y),
$$

in $D$ dimensions. If we denote by $f$ the Fourier transform of the test function, we can further write $\varphi(x)$ in terms of creation and destruction operators, leading to

$$
\varphi(x)=\int \frac{d^{D-1} \mathbf{p}}{(2 \pi)^{D-1}} \frac{f\left(\varepsilon_{p}^{2}, \mathbf{p}^{2}\right)}{2 \varepsilon_{p}}\left[a_{\mathbf{p}}^{\dagger} e^{i p . x}+a_{\mathbf{p}} e^{-i p \cdot x}\right]
$$

with $\varepsilon_{p}^{2}=\mathbf{p}^{2}+m^{2}$.

From this decomposition, it is apparent that test functions should be attached to each fermion and boson fields. Each propagator being the contraction of two fields should be proportional to $f^{2}$. In order to have a dimensionless argument for $f$, we shall introduce an arbitrary scale $\Lambda$ to measure all momenta. $\Lambda$ can be any of the masses of the constituents. To deal with massless theories, we shall consider some a-priori arbitrary value. The final expression of any amplitude should be independent of $\Lambda$.

As recalled in Ref. [3], the test function $f$ should have three important properties:

$i$ ) the physical field $\varphi(x)$ in (2.1) should be independent of the choice of the test function $\rho$. This may be achieved if $f$, the Fourier transform of $\rho$, is chosen among the partitions of unity (PU) (see [3] for more details on PU's). It is a function of finite support which is 1 everywhere except at the boundaries. This choice is also necessary in order to satisfy Poincaré invariance. This is due to the fact that if $f$ is a PU, any power of $f$ is also a PU. In the limit where the test function goes to 1 over the whole space, we then have $f^{n} \rightarrow f$ and Poincare invariance is thus recovered.

ii) In order to be able to treat in a generic way singular distributions of any type, the test function is chosen as a super regular test function (SRTF). It is a function of finite extension - or finite support - vanishing, as well as all its derivatives, at its boundaries, in the UV and in the IR domains.

iii) The boundary conditions of the test function - which in this study is assumed to depend on a one dimensional variable $X$ - should embody a scale invariance inherent, in the UV domain for instance, to the limit $X \rightarrow \infty$ since in this limit $\eta^{2} X$ also goes to $\infty$, where $\eta^{2}$ is an arbitrary dimensionless scale. This can be done by considering a running boundary condition for the test function, i.e. a boundary condition which depends on the variable $X$ according to

$$
f(X \geq H(X))=0 \quad \text { for } \quad H(X) \equiv \eta^{2} X g(X) .
$$


This condition defines a maximal value, $X_{\max }$, with $f\left(X_{\max }\right)=0$. In order to extend the test function to 1 over the whole space, we shall consider a set of functions $g(X)$, denoted by $g_{\alpha}(X)$, where by construction $\alpha$ is a real positive number smaller than 1. A typical example of $g_{\alpha}(X)$ is given in Ref. [3], where it is shown that in the limit $\alpha \rightarrow 1^{-}$, with $\eta^{2}>1$, the running support of the PU test function then stretches over the whole integration domain, $X_{\max } \rightarrow \infty$ and $f \rightarrow 1$. In this limit $g_{\alpha}(X) \rightarrow 1^{-}$. This running condition is equivalent to having an ultra-soft cut-off [3], i.e. an infinitesimal drop-off of the test function in the asymptotic limit, the rate of drop-off being governed by the arbitrary scale $\eta^{2}$. A similar scale invariance is also present in the IR domain, when $X \rightarrow 0$.

With these properties, the TLRS can be summarized as follows, first in the UV domain. Starting from a general amplitude $\mathscr{A}$ written for simplicity in a one dimensional space as

$$
\mathscr{A}=\int_{0}^{\infty} d X T^{>}(X) f(X)
$$

where $T^{>}(X)$ is a singular distribution, we apply the following general Lagrange formula to $f(X)$, after separating out an intrinsic scale $a$ from the (running) dynamical variable $X$

$$
f^{>}(a X)=-\frac{X}{a^{k} k !} \int_{a}^{\infty} \frac{d t}{t}(a-t)^{k} \partial_{X}^{k+1}\left[X^{k} f^{>}(X t)\right] .
$$

This Lagrange formula is valid for any order $k$, with $k>0$, since $f$ is chosen as a SRTF and it is therefore equal to its Taylor remainder for any $k$. After integration by part in (2.4), and using (2.5), we can thus express the amplitude $\mathscr{A}$ as

$$
\mathscr{A}=\int_{0}^{\infty} d X \widetilde{T}^{>}(X) f(X)
$$

where $\widetilde{T}^{>}(X)$ is the so-called extension of the singular distribution $T^{>}(X)$. In the limit $f \rightarrow 1$, it is given by [2]

$$
\widetilde{T}^{>}(X) \equiv \frac{(-X)^{k}}{a^{k} k !} \partial_{X}^{k+1}\left[X T^{>}(X)\right] \int_{a}^{\eta^{2}} \frac{d t}{t}(a-t)^{k} .
$$

The value of $k$ in (2.7) corresponds to the order of singularity of the original distribution $T^{>}(X)$ [2]. In practice, it can be chosen as the smallest integer, positive or null, which leads to a non singular extension $\widetilde{T}^{>}(X)$. If in the absence of the test function $T^{>}(X)$ leads to a logarithmic divergence in (2.4), $k$ is 0 . It is 1 if the divergence is quadratic. With this choice for $k$, the extension of $T^{>}(X)$ is no longer singular due to the derivatives in (2.7), so that we can safely perform the limit $f \rightarrow 1$ in (2.6), and obtain

$$
\mathscr{A}=\int_{0}^{\infty} d X \widetilde{T}^{>}(X)
$$

which is well defined but depends on the arbitrary dimensionless scale $\eta^{2}$. This scale is the only remnant of the presence of the test function. Note that we do not need to know the explicit form of the test function in the derivation of the extended distribution $\widetilde{T}^{>}(X)$. We only rely on its mathematical properties and on the running construction of the boundary conditions.

The extension of singular distributions in the IR domain can be done similarly [2, 3]. For an homogeneous distribution in one dimension, with $T^{<}[X / t]=t^{k+1} T^{<}(X)$, the extension of the 
distribution in the IR domain is given by

$$
\widetilde{T}^{<}(X)=(-1)^{k} \partial_{X}^{k+1}\left[\frac{X^{k+1}}{k !} T^{<}(X) \ln (\tilde{\eta} X)\right],
$$

with $\tilde{\eta}=\eta^{2}-1$. The usual singular distributions in the IR domain are of the form $T^{<}(X)=$ $1 / X^{k+1}$. In that case $\widetilde{T}^{<}(X)$ reads

$$
\widetilde{T}^{<}(X)=\frac{(-1)^{k}}{k !} \partial_{X}^{k+1} \ln (\tilde{\eta} X)
$$

where the derivative should be understood in the sense of distributions. Doing this, the extension $\widetilde{T}^{<}(X)$ is nothing else than the pseudo-function (Pf) of $1 / X^{k+1}[2,3,9]$

$$
\widetilde{T}^{<}(X)=\operatorname{Pf}\left(\frac{1}{X^{k+1}}\right) .
$$

The extension $\widetilde{T}^{<}(X)$ differs from the original distribution $T^{<}(X)$ only at the $X=0$ singularity.

\section{Application to the fine-tuning problem}

After the electro-weak symmetry breaking as occured, the Higgs couples, among others, to itself, to the leptons and to the gauge bosons. We then consider radiative corrections implying the top quark, the $\mathrm{W}$ and $\mathrm{Z}$ bosons and, as we shall be working in the Feynman gauge, the Goldstone bosons and the Fadeev-Popov ghosts as well. Each of this 1PI contributions gives a contribution to the self-energy $-i \Sigma\left(p^{2}\right)$, where $p$ is the four-momentum of the external particle, and we have

$$
M_{H}^{2}=M_{0}^{2}+\Sigma\left(M_{H}^{2}\right) .
$$

When calcuted using a sharp cut-off procedure, the radiative corrections have divergent terms. We shall concentrate on the terms proportional to $\Lambda_{C}^{2}$ as they are the ones leading to the fine-tuning problem. The full calculation yields

$$
M_{H}^{2}=M_{0}^{2}+\frac{3 \Lambda_{C}^{2}}{8 \pi^{2} v^{2}}\left[M_{H}^{2}+2 M_{W}^{2}+M_{Z}^{2}-4 m_{t}^{2}\right]+\ldots,
$$

where $m_{t}, M_{W, Z}$ and $M_{H}$ are the masses of the top quark, $W, Z$ and Higgs bosons respectively, and $v$ is the vacuum expectation value of the Higgs potential in the Standard Model. The dots include logarithmic corrections in $\Lambda_{C}$ as well as contributions independent of $\Lambda_{C}$ in the large $\Lambda_{C}$ limit.

As mentionned before, when using a cut-off procedure, we should have, from consistency arguments, $M_{H}^{2}<\Lambda_{C}<\Lambda_{e f f}$. We would also like $\Lambda_{C}$ to be close to $\Lambda_{e f f}$ so that amplitudes will be more likely to be independent of its precise value. The fine-tuning issue then appears when one requires that $M_{H}^{2}$ should be defined without any large cancellation taking place in (3.2). The degree of fine-tuning then depends explicitly on how large $\Lambda_{C}$ is. Furthermore, in the litterature, the mathematical scale $\Lambda_{C}$ is often taken equal to the physical one $\Lambda_{\text {eff }}$. Following this identification, the problem gets worse because one would like, at the same time, $\Lambda_{\text {eff }}$ to be small, to reduce the fine-tuning, and big to get a proper physical description. The relation (3.2) then becomes a test ground for "new physics" theory, provided it predicts a value of $M_{H}^{2}$ and $\Lambda_{e f f}$, by evaluating the 


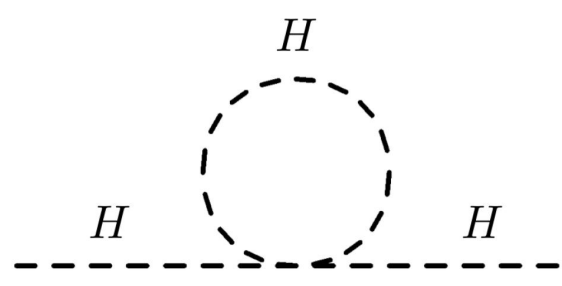

Figure 1: The $\Sigma_{1 b, H}$ Higgs loop contribution

amount of fine-tuning it implies. The fine-tuning process then offers a way to supposedly safe and time-lasting predictions on the onset of "new physics" beyond the standard model [10].

We now turn to the computation of the radiative corrections using the TLRS. As mentioned in the previous section, all amplitudes turn out to be finite. We will illustrate it by detailling the calculation of the simple Higgs loop contribution in Fig. 1. In Euclidean space one has

$$
-i \Sigma_{1 b, H}=-\frac{3 i M_{H}^{2}}{2 v^{2}} \int_{0}^{\infty} \frac{d^{4} k_{E}}{(2 \pi)^{4}} \frac{1}{k_{E}^{2}+M_{H}^{2}} f\left(\frac{k_{E}^{2}}{\Lambda^{2}}\right),
$$

where $k_{E}^{2}$ is the square of the four-momentum $k$ in Euclidean space. As already mentioned in Sec. 2, $\Lambda$ is an arbitrary momentum scale. The test function $f$ provides the necessary (ultra-soft) cut-off in the calculation of the integral.

After an evident change of variable, we get

$$
\begin{aligned}
\Sigma_{1 b, H} & =\frac{3 M_{H}^{4}}{32 \pi^{2} v^{2}} \int_{0}^{\infty} d X \frac{X}{X+1} f\left(\frac{M_{H}^{2}}{\Lambda^{2}} X\right) \\
& =\frac{3 M_{H}^{4}}{32 \pi^{2} v^{2}} \int_{0}^{\infty} d X\left(1-\frac{1}{X+1}\right) f\left(\frac{M_{H}^{2}}{\Lambda^{2}} X\right) .
\end{aligned}
$$

The first term under the integral can be reduced to a pseudo-function, using (2.10). Indeed, with $Z=1 / X$, we have

$$
\begin{aligned}
\int_{0}^{\infty} d X f(X) & =\int_{0}^{\infty} \frac{d Z}{Z^{2}} f\left(\frac{M_{H}^{2}}{\Lambda^{2}} \frac{1}{Z}\right) \\
& =\int_{0}^{\infty} d Z \operatorname{Pf}\left(\frac{1}{Z^{2}}\right) \\
& =-\left.\frac{1}{Z}\right|^{\infty}=0
\end{aligned}
$$

The notation $\left.f(u)\right|^{a}$ simply indicates that $f(u)$ should be taken at the value $u=a$, the lower limit of integration being taken care of by the definition of the pseudo-function. The self-energy thus writes

$$
\Sigma_{1 b, H}=-\frac{3 M_{H}^{4}}{32 \pi^{2} v^{2}} \int_{0}^{\infty} d X \frac{1}{X+1} f\left(\frac{M_{H}^{2}}{\Lambda^{2}} X\right) .
$$

The constant factor $M_{H}^{2} / \Lambda^{2}$ in the argument of the test function has no physical meaning since it can be absorbed by a rescaling of the arbitrary dimensionless scale $\eta^{2}$. This can be easily seen 
by applying the Lagrange formula (2.5) with the intrinsic scale $a=M_{H}^{2} / \Lambda^{2}$ and $k=0$. It can thus safely be removed ${ }^{1}$.

We can now apply the Lagrange formula for $k=0$. Using the boundary condition on the support of the test function

$$
X t \leq H(X)=\eta^{2} X g_{\alpha}(X),
$$

we finally get, in the limit $f \rightarrow 1$

$$
\begin{aligned}
\Sigma_{1 b, H} & =-\frac{3 M_{H}^{4}}{32 \pi^{2} v^{2}} \int_{0}^{\infty} d X \partial_{X}\left(\frac{X}{X+1}\right) \int_{1}^{\eta^{2}} \frac{d t}{t} \\
& =-\frac{3 M_{H}^{4}}{32 \pi^{2} v^{2}} \ln \left(\eta^{2}\right) .
\end{aligned}
$$

It is easy to see that using a naïve cut-off on $k_{E}^{2}$ one would have obtained, in the large $\Lambda_{C}$ limit

$$
\Sigma_{1 b, H}^{C}=\frac{3 M_{H}^{2}}{32 \pi^{2} v^{2}}\left[\Lambda_{C}^{2}-M_{H}^{2} \ln \left(\frac{\Lambda_{C}^{2}}{M_{H}^{2}}\right)\right] .
$$

The quadratic and logarithmic divergent terms using a cut-off procedure are transmuted in TLRS into contributions depending on the arbitrary dimensionless scale $\eta^{2}$.

The other contributions to the radiative corrections to the Higgs mass can be calculated similarly. The final correction to the bare mass can thus be written schematically as

$$
M_{H}^{2}=M_{0}^{2}+\bar{a} \eta^{2}+\bar{b} \ln \left[\eta^{2}\right]+c t e .
$$

This should be compared with the corrections indicated in Eq. (3.2) when using a naive cut-off. The exact expression of $\bar{a}$ is of no physical interest since physical observables should be RGindependent of $\eta^{2}$. They are completely finite and depend only on the masses of the top quark, $W, Z$ and Higgs bosons (and on the vacuum expectation value $v$ of the Higgs field).

As it is, this equation has not much physical interest. It is just a definition of the bare mass as a function of the physical mass, in the spirit of the renormalization theory. Away from the on mass shell condition $p^{2}=M_{H}^{2}$ however, the constant term includes $p^{2}$-dependent logarithmic corrections which give rise to the well-known running of the mass. These corrections are identical in both regularization schemes, as expected.

One can easily see that no fine-tuning problem arises when using TLRS as no other mass scales than the constituent masses enter the calculation. However, amplitudes are function of the dimensionless scale $\eta^{2}$ in a similar fashion that dimensional regularized amplitudes depend on $\frac{\mu^{2}}{M^{2}}$ [7]. The fine-tuning problem using the BPHZ scheme as been analyzed long ago in [11] yelding similar conclusions.

\section{Conclusion}

In this work we have calculated the radiative corrections to the Higgs mass to the first loop order using alternatively a cut-off regularisation and the TLRS. Results appear to differ significantly.

\footnotetext{
${ }^{1}$ This could also be done more directly by choosing a particular value for $\Lambda$.
} 
The cut-off result exihibits a quadratic dependence in the cut- off scale $\Lambda_{C}$, so that under certain requirements it is the source of the fine-tuning issue. This issue get worsened when $\Lambda_{C}$ is identified with $\Lambda_{e f f}$. In turn, when using the TLRS, the amplitudes are finite and, apart from the constituent masses, they only exhibit a dependence on the arbitrary scale $\eta$, thus preventing any fine-tuning problem from arising. A further analysis could be carried in the spirit of effective field theories by limiting the stretching of the test function's support to a finite value defined by $\Lambda_{C}$. This will be detailled in a future paper.

\section{References}

[1] M. Kaku, Quantum Field Theory: A Modern Introduction, Oxford University Press, 1993

[2] P. Grangé and E. Werner, Quantum fields as Operator Valued Distributions and Causality, arXiv: math-ph/0612011 and Nucl. Phys. B, Proc. Supp. 161 (2006) 75.

[3] P. Grangé, J.-F. Mathiot, B. Mutet and E. Werner, Taylor-Lagrange renormalization scheme: Application to light-front dynamics, Phys. Rev. D80 (2009)105012; Taylor-Lagrange renormalization scheme, Pauli-Villars subtraction, and light-front dynamics, Phys. Rev. D82 (2010) 025012.

[4] H. Epstein and V. Glaser, The role of locality in perturbation theory, Ann. Inst. Henri Poincaré, XIX A (1973) 211.

[5] J.M. Gracia-Bondia, Improved Epstein-Glaser Renormalization in Coordinate Space I. Euclidean Framework, Math. Phys. Anal. Geom. 6 (2003) 59;

J.M. Gracia-Bondia and S. Lazzarini, Improved Epstein-Glaser renormalization. II. Lorentz invariant framework, J. Math. Phys. 44 (2003) 3863.

[6] S.S. Schweber, An Introduction to Relativistic Quantum Field Theory, Harper and Row, 1964, p.721. J. Collins, Renormalization, Cambridge University Press, 1984, p.4.

R. Haag, Local Quantum Physics: Fields, Particules, Algebras, Texts and Monographs in Physics, Springer-Verlag, Berlin 1996,2nd Edition.

[7] B. Mutet, P. Grange and E. Werner Taylor-Lagrange Renormalisation: Self-energy of the gauge boson, in proceedings of LC2010 Relativistic nuclear and particle physics, POS (LC2010) 026.

[8] G. Scharf, Finite QED: the causal approach, Springer Verlag, Berlin 1995.

A. Aste, Finite field theories and causality, in proceedings of LC2008 Relativistic nuclear and particle physics, POS (LC2008) 001, and references therein.

R. Stora, Lagrangian field theory, in proceedings of Les Houches Summer School, Session 21, C. DeWitt-Morette and C. Itzykson eds., Gordon and Breach 1973.

[9] L. Schwartz, Théorie des distributions, Hermann, Paris 1966.

[10] S. Cassel, D.M. Ghilencea and G.G. Ross, Fine tuning as an indication of physics beyond the MSSM, Nuc. Phys. B 825 (2010) 203.

[11] O.E. Foda, The fine-tuning problem in renormalized perturbation theory: Spontaneously-broken gauge models, Phys. Lett. B 124 (1983) 192. 